\title{
Implant Placement Using Alveolar Ridge Split in Atrophic Maxillary Alveolar Bone
}

\author{
Youngdeok Chee, DDS, MSD, PhD* \\ Professor, Department of Oral \& Maxillofacial Surgery, College of Dentistry, Wonkwang University, Iksan, Korea \\ *Corresponding author: Youngdeok Chee, Department of Oral \& Maxillofacial Surgery, Sanbone dental \\ hospital, College of Dentistry, Wonkwang University, 1142, Sanbon-dong, Gunpo-city, Gyeonggi-do \\ 15865 , Korea. \\ Tel:+82-31-390-2875. Fax : +82-31-390-2777. E-mail : omschee@wku.ac.kr
}

\section{OPEN ACCESS}

pISSN 1229-5418

eISSN 2671-6623

Implantology 2020; 24(4): 193-202

https://doi.org/10.32542/implantology.202017

Received: August 13, 2020

Revised: September 26, 2020

Accepted: October 21, 2020

ORCID

Youngdeok Chee

https://orcid.org/0000-0002-0333-4685

Copyright $($ 2020. The Korean Academy of Oral \& Maxillofacial Implantology

\footnotetext{
This is an Open Access article distributed under the terms of the Creative Commons Attribution Non-Commercial License (http://creativecommons. org/licenses/by-nc/4.0/) which permits unrestricted non-commercial use, distribution, and reproduction in any medium, provided the original work is properly cited.
}

\begin{abstract}
If the volume of the horizontal alveolar bone is insufficient during the implant procedure, it is difficult to establish a fixture of the required diameter without surgical procedures such as block bone graft, lateral bone graft, and ridge split. Among them, alveolar ridge split is known as an effective technique to increase the volume of the horizontal alveolar bone using maxillary bone elasticity. The theoretical background of this technique is to provide a basis for maintaining the implant with the expectation that new bone will form when the implant and bone graft material are operated, forming a space surrounded by the bone and periosteum. In two cases reported herein, there was no postoperative inflammatory reaction and normal healing was observed. Since then, the follow-up radiograph has shown good outcomes, and ongoing progress is being observed. Alveolar ridge split and bone graft are effective techniques for horizontal alveolar augmentation. In these cases, it was actually possible to form a stable alveolar ridge for implant placement through a successful procedure in the maxillary edentulous region.
\end{abstract}

Keywords: Alveolar ridge split, Dental implant, Guided bone regeneration

\section{I . Introduction}

If a tooth is extracted, the maxillary anterior alveolar bone does not receive physiological stimulus and is subsequently absorbed and atrophied rapidly. In particular, the thin buccal cortical bone is absorbed, and as a result, the maxillary anterior alveolar bone is atrophied and thinned to the extent that implant placement is difficult. The causes of absorption include disuse atrophy, denture pressure and aging. However, types of absorption vary depending on the thickness of the cortical bone, muscle attachment and the direction of bite force for each part of maxilla and mandible, and in general, if eventually the alveolar crest is tapered and the absorption of the alveolar bone continues, the alveolar crest is gradually lowered, and the occlusal vertical dimension is reduced, leading to 
mandibular prognathic malocclusion.

Particularly, since the absorption of the buccal cortical bone is more severe than the palate in the maxilla, the arch becomes narrower and appears receding. In contrast, in the mandible, as the buccal compact bone is thick and resists absorption, and the occlusal vertical dimension decreases, the mandible rotates and is placed in front of the maxillary bone.

Methods for lateral bone augmentation of such an atrophic maxillary alveolar bone include guided bone regeneration, block bone graft and alveolar ridge split technique. Alveolar ridge split technique was first introduced by Simion ${ }^{1}$ in 1992, in which split-crest technique and guided tissue regeneration were used in the receding maxillary bone. Since then, the procedure has been widely known and is mostly used for the purpose of buccolingual alveolar bone augmentation and aesthetic restoration in the maxillary anterior teeth.

Alveolar ridge split uses the elasticity of the alveolar bone to move the cortical bone in the buccal direction, recovering the atrophic buccal bone, which enables the implant placement and aesthetic bone formation of maxillary anterior teeth. ${ }^{2}$ In addition, since the implant is placed between the buccal and cortical bones and bone graft is carried out, blood supply between two bone plates is sufficient, and since healing is made as if after the tooth extraction, the new bone of good quality is generated. As a result, the osseointegration of the implant is facilitated. ${ }^{3}$ Another advantage of alveolar ridge split is that a single surgery can increase the width of the alveolar bone and simultaneously place the implant, which reduces the overall surgery time. Furthermore, the generated bone in this surgery has low absorption rates, leading to high long-term success rates of implant. Thus, as we had used alveolar ridge split to increase the width of the alveolar bone and simultaneously to place the implant on the absorbed and atrophic maxillary alveolar bone, and obtained good outcomes, herein we report the cases.

\section{II . Case Report}

\section{Case 1}

A 71-year old male patient visited the clinic for the purpose of implants in both maxilla and mandible. In the Department of Prosthesis, a plan for extraction of 4 residual maxillary teeth and placement of 4 implants followed by implant-retaining denture was established (Fig. 1A). Residual teeth including both maxillary central incisors and maxillary right lateral incisor were extracted, and implant placement surgery accompanied by alveolar ridge split and guided bone regeneration was planned (Fig. 1B).

After a month from the tooth extraction, the maxillary anterior region was healed well, and a local 
anesthesia (2\% lidocaine with 1:100,000 epinephrine, Yuhan, Korea) was performed (Fig. 2A). Single vertical incision was performed on each of the center of the Maxilla and the left and right posterior regions to form a flap, and after horizontal incision connected to the vertical incisions, the flap was elevated (Fig. 2B). After the elevation of the flap, extraction sockets were observed in the anterior regions of the maxilla. For bone graft and implant placement, after vertical and lateral osteotome was performed on the buccal bone plate of the receding maxillary alveolar bone using a chisel for alveolar ridge splitting (Gene chisel ${ }^{\circledR}$, Stoma, German), and the maxillary buccal bone plate was expanded in the buccal direction by $4-5 \mathrm{~mm}$ (Fig. 2C). Because the bevel of the chisel used is gently sloped, the bone does not expand rapidly, so the gap can be widened to prevent the fracture.

The region between the maxillary buccal and palatine bone plates was expanded carefully not to break the expanded buccal bone plate, and subsequently, implant drilling was performed, followed by placement of the implant (Implantium ${ }^{\circledR}$, Dentium, Korea) (Fig. 2D).
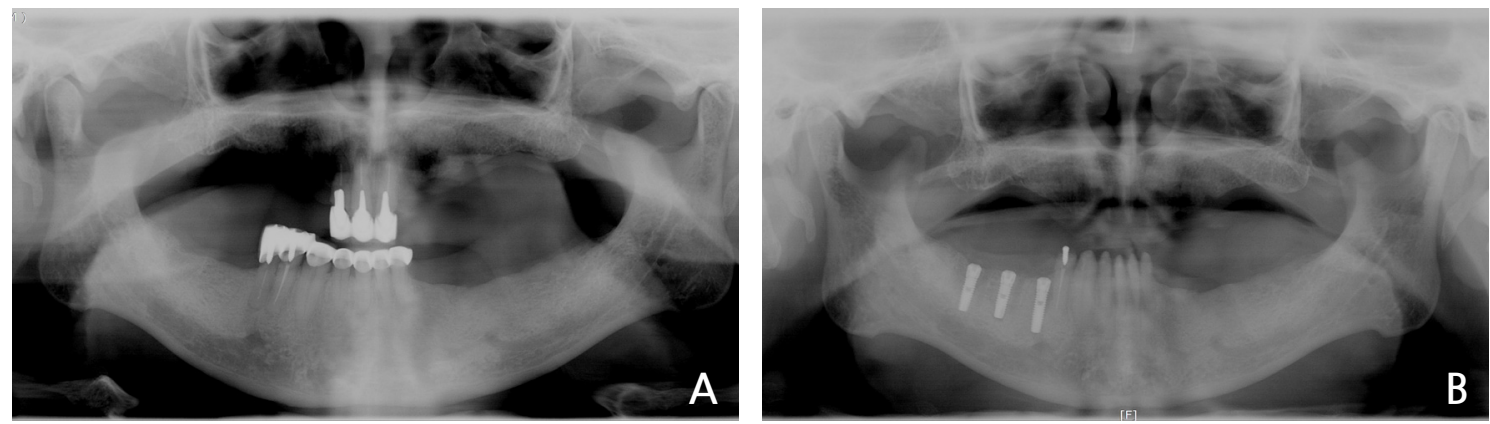

Fig. 1. Pre-operative panoramic view. (A) First visit, (B) Maxillary residual teeth were extracted.
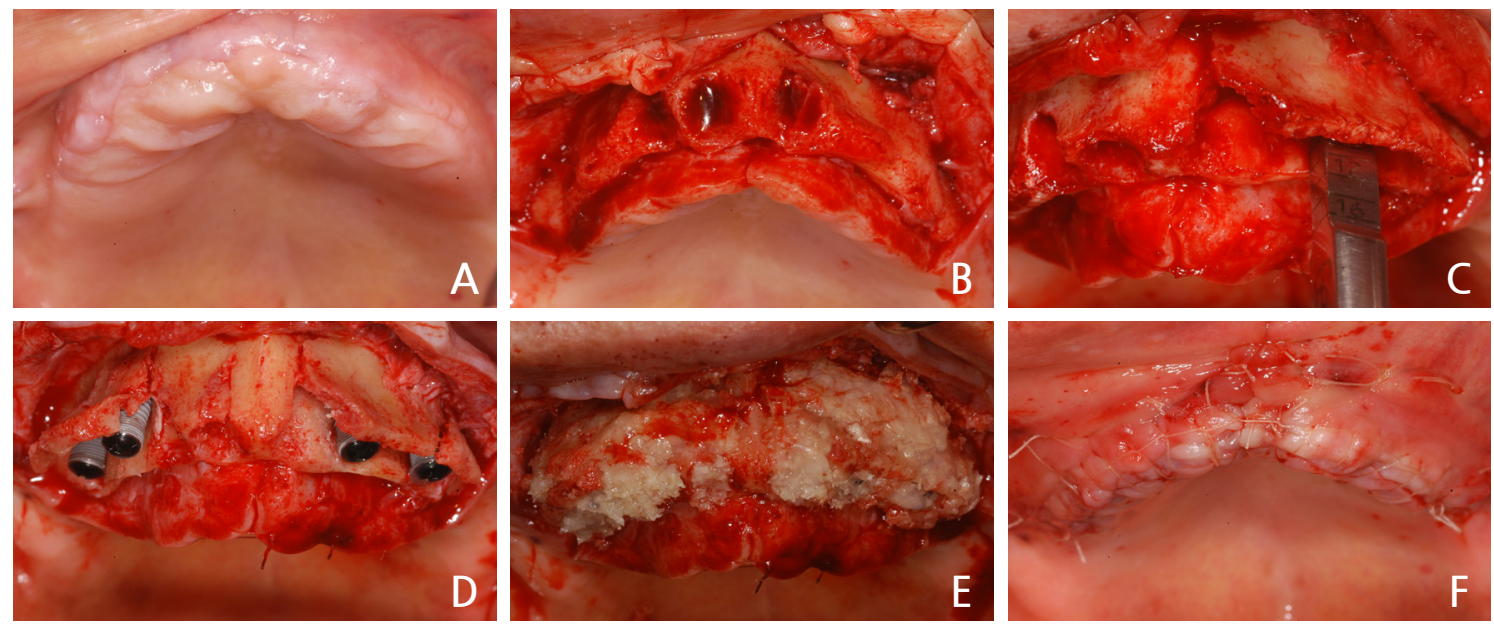

Fig. 2. Intraoral views of alveolar ridge split technique with guided bone regeneration. (A) Pre-operative clinical gingiva, (B) Full thickness flap was elevated, (C) Alveolar bones were split and spread apart with a chisel, (D) Implants were inserted, (E) Defects were augmented with granules of allograft, (F) The flap was sutured without tension. 
After the placement of the implant, bone graft was performed on the space between the maxillary buccal and palatine bone plates and the defects using allogenic bone (Puros ${ }^{\circledR}$, Zimmer Biomet, USA), and guided bone regeneration performed in parallel using an absorbable membrane (Lyoplant ${ }^{\circledR}$, B.Braun, Germany) (Fig. 2E). The periosteal releasing incision was performed on the buccal flap, and sutured without tension by the single interrupted suture and horizontal mattress suture (Fig. 2F).

After surgery, panorama radiograph was taken, which showed bone graft and implant placement through alveolar ridge split had been performed well (Fig. 3).

After 7 months from the surgery, the surgical site had been healed well without the implant exposure, and a local anesthesia was performed for the second surgery (Fig. 4A). Single vertical incision was

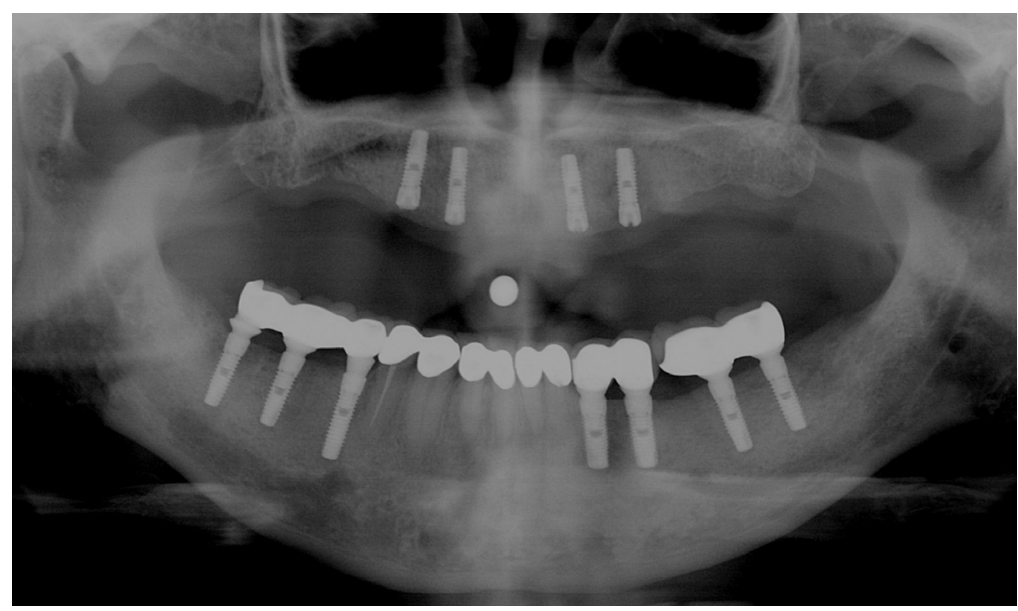

Fig. 3. Panoramic view after alveolar ridge split \& implant placement.
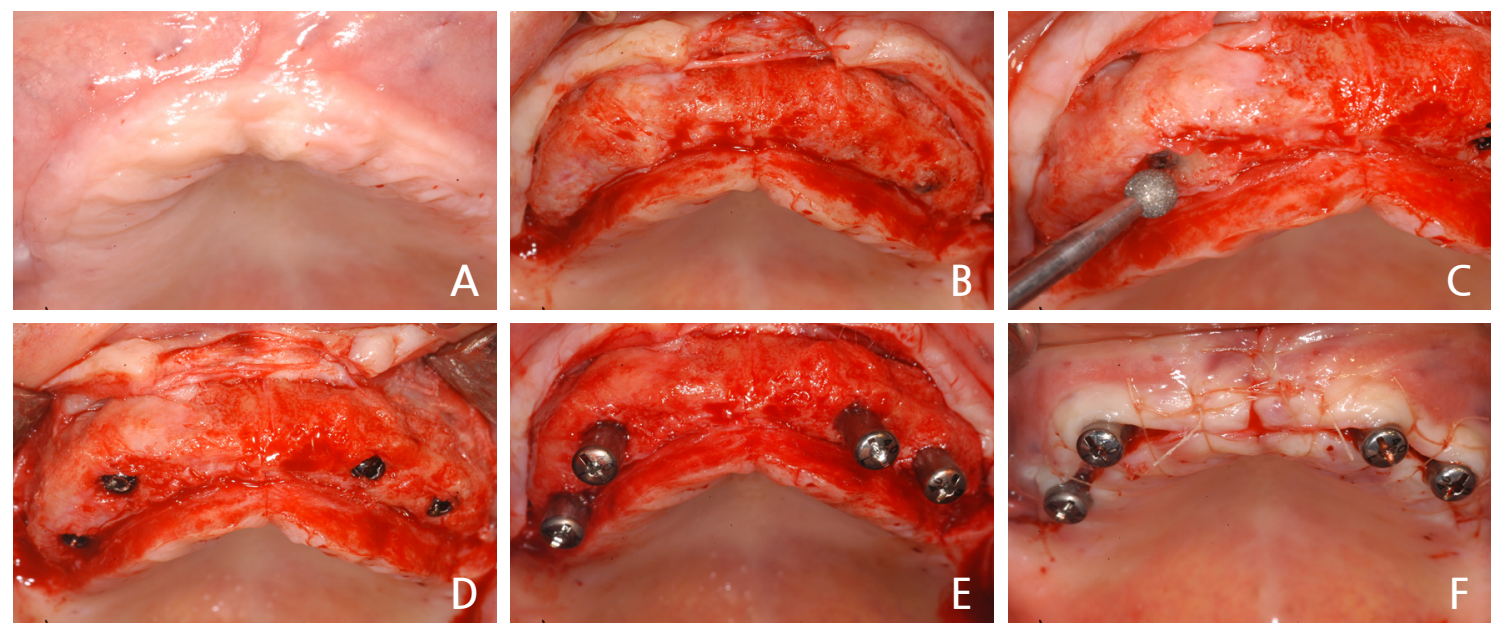

Fig. 4. Intraoral views of implant second surgical procedure. (A) Gingival condition before second surgery, (B) Full thickness flap was elevated, (C) The alveolar bone was ground with a diamond round bur, (D) The cover screws were exposed, $(E)$ Healing abutments were placed on the implant, $(F)$ The flap was sutured. 


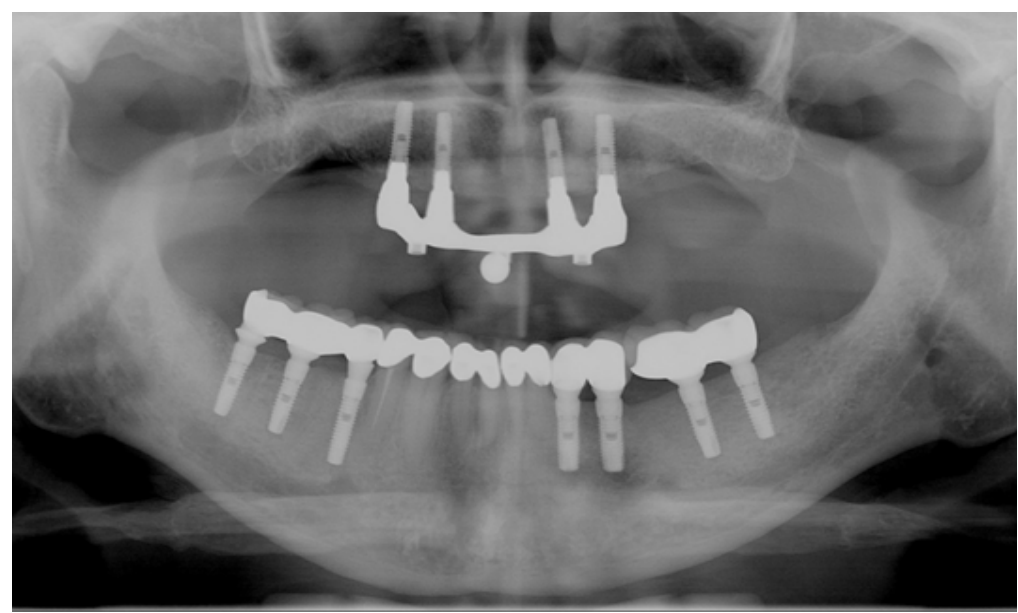

Fig. 5. Panoramic view after final restoration setting.

performed on each of the center of the maxilla and the left and right posterior regions just as in the first surgery, and after the horizontal incision performed on the alveolar crest, the full-thickness flap was elevated (Fig. 4B). As new bone of good quality had been generated on the surgical sites of alveolar ridge split and bone graft, and implant placement sites were also covered with bone, the bone was removed with a diamond round bur (Fig. 4C) to expose the cover screws (Fig. 4D). After exposure of the cover screws, healing abutments were assembled onto them (Fig. 4E), and the flap was sutured with the single interrupted suture (Fig. 4F). After treatment of soft tissue, the implant retaining denture was fabricated through the production of a bar structure (Fig. 5).

\section{Case 2}

A 39-year old female patient visited the clinic with the complain of a loose prosthesis, and upon examination, the secondary caries was discovered around the abutment of the maxillary prosthesis. Thus, the extraction of the decayed teeth and the implant placement along with alveolar ridge split were planned (Fig. 6A).

After removal of the prosthesis, canines on both sides of the maxilla, which only had their root due to caries, were extracted (Fig. 6B), and after 3 weeks from the tooth extraction, a local anesthesia (2\% lidocaine with 1:100,000 epinephrine, Yuhan, Korea) was performed for bone graft and implant placement (Fig. 7A). Vertical incision and horizontal incision were performed to elevate a flap (FIg. 7B), and after vertical and horizontal osteotome had been performed using a chisel (Gene chisel ${ }^{\circledR}$, Stoma, German), the buccal cortical bone plate was expanded in the buccal direction by $4 \mathrm{~mm}$ to perform bone graft through alveolar ridge split (Fig. 7C). After the bone plate had been expanded in the buccal direction 
through alveolar ridge split, drilling was performed beneath the expanded region, and the implant (Xive ${ }^{\circledR}$, Dentsply, USA) was placed there (Fig. 7D). Following implant placement, the expanded bone region and defects underwent bone graft using a mixture of allogenic bone (ICB ${ }^{\circledR}$, Rocky Mountain Tissue Bank, USA) and bone substitute (Osteon ${ }^{\circledR}$, Genoss, Korea), and were covered with an absorbable membrane (Bio-arm ${ }^{\circledR}$, ACE surgical supply Co., USA) (Fig. 7E). After bone graft, the periodontal flap was sutured without tension by the single interrupted suture and horizontal mattress suture using the periosteal releasing incision (Fig. 7F). On post-surgery panorama radiograph, it could be seen that bone graft and implant placement through alveolar ridge split had been performed well (Fig. 8).

After 6 months from the surgery, it was observed that surgical sites were healed well, and a local anesthesia was performed for the second surgery (Fig. 9A). A flap for the second surgery was designed in the form of Z-plasty for the aesthetics of the maxillary anterior teeth (Fig. 9B). The flap was shaped
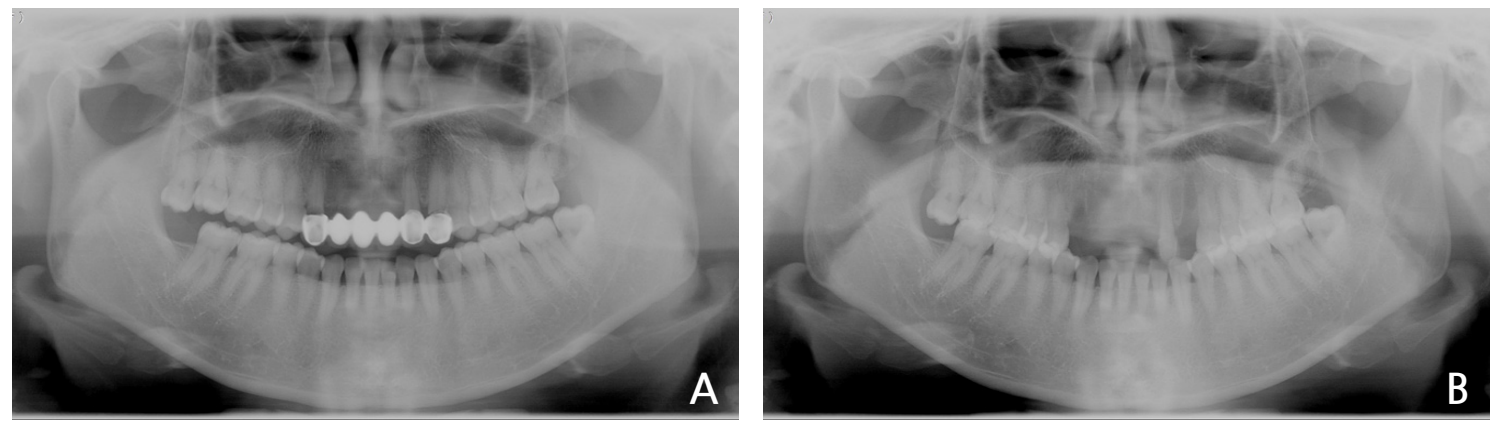

Fig. 6. Pre-operative panoramic view. (A) First visit, (B) Maxillary teeth with poor prognosis were extracted.
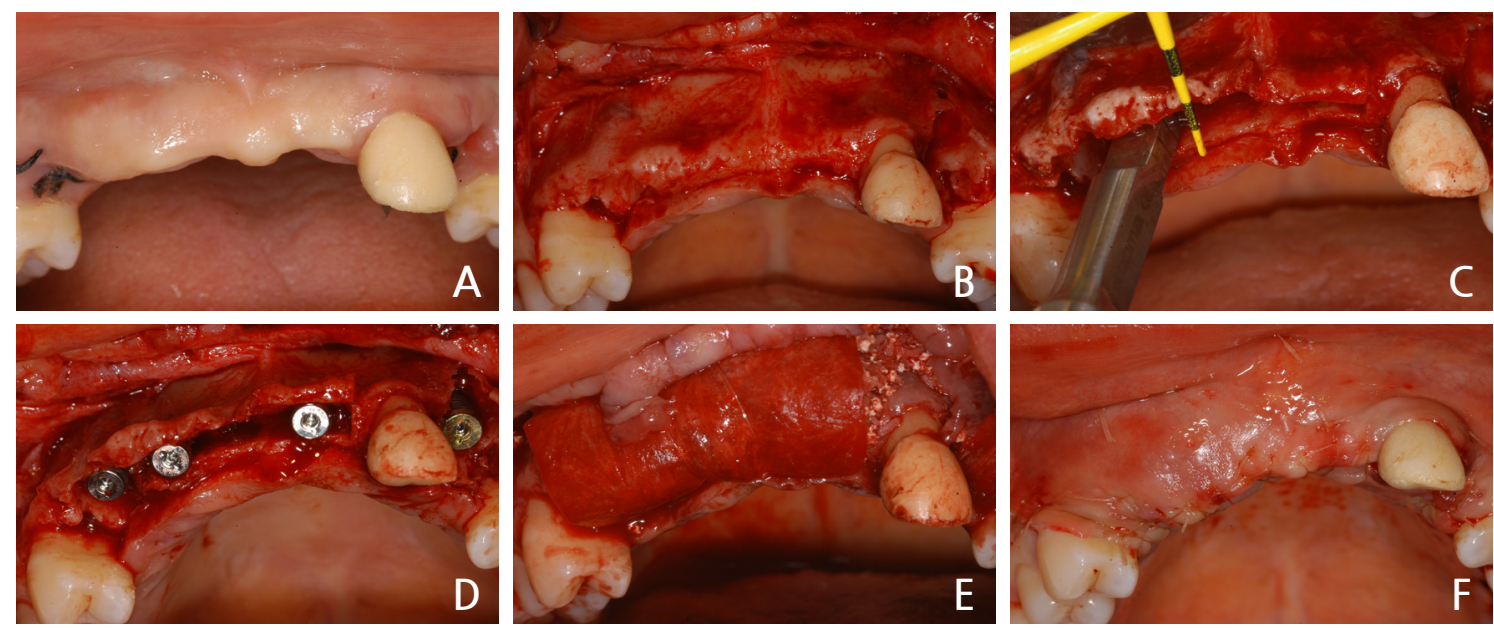

Fig. 7. Intraoral views of alveolar ridge split technique with guided bone regeneration. (A) Pre-operative clinical gingiva, (B) Full thickness flap was elevated, (C) Alveolar bones were split and spread apart with a chisel, (D) Implants were inserted, (E) Defects were augmented with granules of a mixture of allograft and alloplast, (F) The flap was sutured without tension. 
to fit the placement site and elevated (Fig. 9C), and after healing abutments had been connected (Fig. 9D), it was sutured with the single interrupted suture (Fig. 9E). After treatment of soft tissue, the final fixed-type prostheses for the maxillary anterior implant was fabricated (Fig. 9F). The panorama radiograph taken following the installation of the final prostheses showed that the state of bone graft and implant placement were observed to be excellent (Fig. 10), and the panorama radiograph taken after 13 years from the installation of the prostheses also showed that the sites of bone graft and the implant prostheses were maintained satisfactorily (Fig. 11).

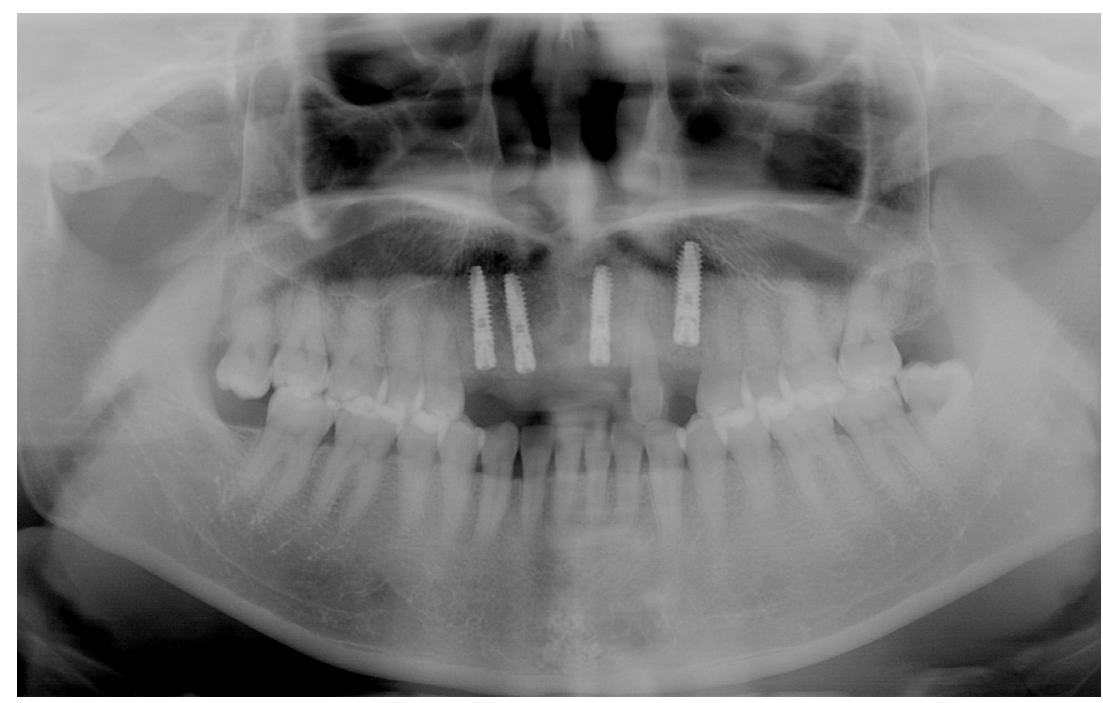

Fig. 8. Panoramic view after alveolar ridge split \& implant placement.
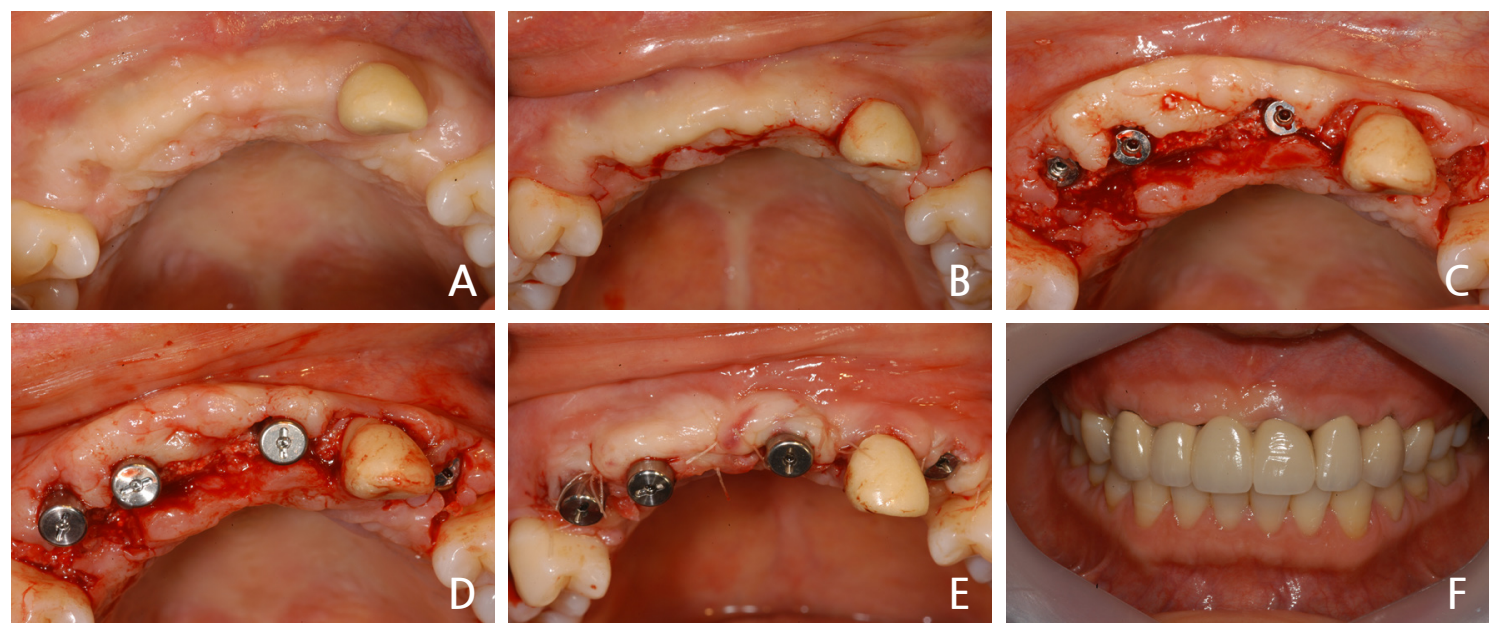

Fig. 9. Intraoral views of implant second surgical procedure and final restoration. (A) Gingival condition before second surgery, (B) Flap design with Z-plasty, (C) Full thickness flap was elevated and cover screws were exposed, (D) Healing abutments were placed on the implant, (E) The flap was sutured, (F) Final prosthesis inserted state. 


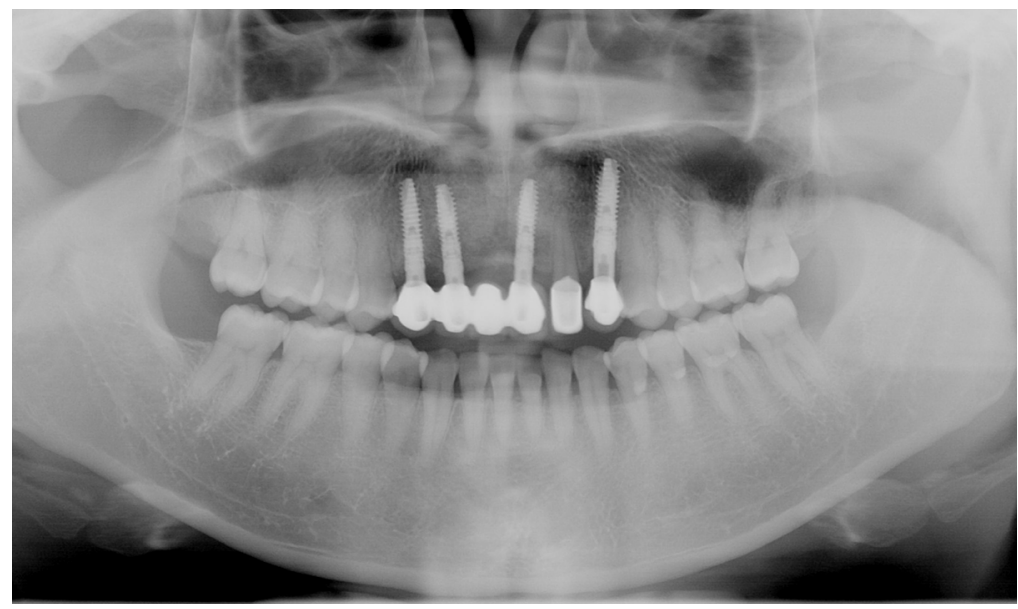

Fig. 10. Panoramic view after final restoration setting.

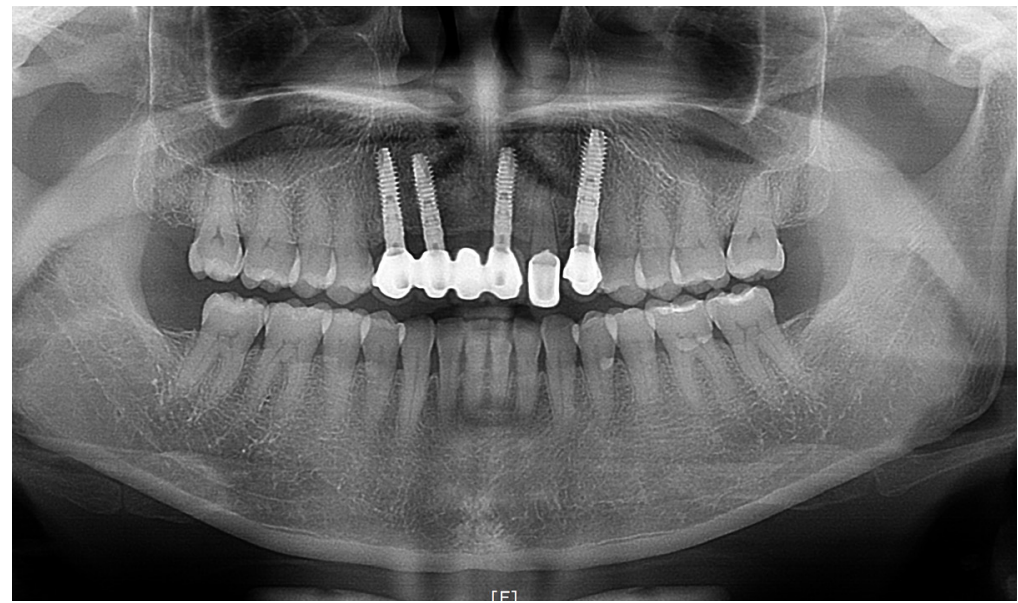

Fig. 11. Panoramic view after 13 years from final restoration.

\section{Discussion}

Alveolar ridge split technique can be used in bone augmentation for implant placement in the maxillary alveolar bone that is sufficiently high but has a thin buccolingual section.

In 2000, Sethi et al. ${ }^{4}$ investigated 150 patients with 449 implant placements by alveolar ridge split for 27 months and reported a 97\% success rate while in 2005, Ferringo et al. ${ }^{5}$ reported a $97.6 \%$ success rate from 82 implant placements in 40 partial edentulous patients using the same technique.

Alveolar ridge split has the advantages that new bone is generated more rapidly and absorption occurs less often than other bone augmentation procedures, and overall treatment time can be reduced since implant placement and bone graft are performed simultaneously by creating a space surrounded by both bone plates for bone augmentation. 
Simion et al. ${ }^{1}$ performed alveolar ridge split on the alveolar bone with sufficient height but narrow width, along with subsequently guided tissue regeneration using a non-absorbable membrane (e-PTFE), and reported that the alveolar bone width could be increased by $3-4 \mathrm{~mm}$ in the maxillary anterior region and $1-1.5 \mathrm{~mm}$ in the mandible.

In these two cases, similar to the Simion's method, alveolar ridge split and guided bone regeneration were performed along with bone graft on the vacant space of the expanded bone plates and the buccal bone defects. The membrane used for this procedure was not a non-absorbable membrane (e-PTFE) used by Simion, but an absorbable membrane that was easy to handle and less exposed.

Scipioni et al. ${ }^{6}$ suggested that the thickness of the buccal bone plate be at least $1.5 \mathrm{~mm}$ after split of the alveolar ridge, and Qahash et al. ${ }^{7}$ reported that the buccal bone with at leat $2 \mathrm{~mm}$ thickness was required when implants were placed in the extraction socket. In the two cases of this study, since the atrophic maxillary alveolar ridge was split and expanded, it was difficult to form the thick bone plates, and the thickness of $1.5 \mathrm{~mm}$ was the thickest that could be maintained. If the maxillary alveolar ridge is thick enough, it may be considered that the implant is placed first, and subsequently guided bone regeneration is performed on the less thick buccal region rather than performing alveolar ridge split technique.

Coatoam et al. ${ }^{8}$ reported that only the bone was grafted in the space between bone plates without placing the implant when fixation was not made correctly or the placement direction was wrong implant placement, and if in doubt about the stability of the bone plate even when the implant was fixed well and the placement direction was right, the bone plates was ligated and fixed with a wire. Shimoyama et al. ${ }^{9}$ suggested that it was better to place a tapered implant and perform bone augmentation simultaneously when an implant was placed using alveolar ridge split in the severely atrophic maxilla.

In the two cases of this study, as in Shimoyama et al., ${ }^{9}$ the tapered implant was also used to achieve good fixation. Since atrophic alveolar ridge was split and expanded, it was difficult to obtain fixation of the implant in the expanded bone plate. For implant fixation, drilling was performed into the basal bone, passing through the split bone plate such that a $2-3 \mathrm{~mm}$ of the tip of the implant could be placed. In both cases, implant placement and alveolar ridge split were performed simultaneously to shorten the overall treatment time for the completion of the implant prosthesis.

It is considered that using a gradually expanding chisel is advantageous to prevent the bone plate from being fractured when alveolar ridge split is attempted. If the bone plate is fractured, it may be considered that only the bone is grafted without implant placement after fixing the bone plate using a wire or small steel plate as done by Coatoam et al., ${ }^{8}$ and the implant is placed 4 to 5 months later.

It is considered that good outcomes can be obtained by performing high-quality new bone 
augmentation and implant placement simultaneously along with alveolar ridge split technique through the development of techniques suitable for various forms of the atrophic alveolar bone and of devices that prevents fracture of the bone plate due to rapid expansion.

\section{IV . Conclusion}

In these clinical cases, rapid bone regeneration and high-quality bone augmentation were achieved using alveolar ridge split technique and guided bone regeneration on the atrophic maxillary alveolar bone, where implant placement is difficult due to the narrow buccolingual section, and implants were placed simultaneously, leading to the shortened treatment time and eventually completing a successful implant placement procedure.

\section{References}

1. Simion M, Baldoni M, Zaffe D. Jawbone enlargement using immediate implant placement associated with a split-crest technique and guided tissue regeneration. Int J Periodontics Restorative Dent 1992;12:462-73.

2. Georg E, Gerd W, Rolf E. Preliminary report on a staged ridge splitting technique for implant placement in the mandible: a technical note. Int J Oral Maxillofac Implants 2006;21:445-9.

3. Calvo Guirado JL, Yuguero MR, Carrion del Valle MJ, Zamora GP. A maxillary ridge-splitting technique followed by immediate placement of implants: a case report. Implant Dent 2005; 14:14-20.

4. Sethi A, Kaus T. Maxillary ridge expansion with simultaneous implant placement: 5-year results of an ongoing clinical study. Int J Oral Maxillofac Implants 2000;15:491-9.

5. Ferrigno N, Laureti M. Surgical advantages with ITI TE implants placement in conjunction with split crest technique. 18-month results of an ongoing prospective study. Clin Oral Implants Res 2005; $16: 147-55$.

6. Scipioni A, Bruschi GB, Calesini G. The edentulous ridge expansion technique: a five-year study. Int J Periodontics Restorative Dent 1994;14:451-9.

7. Qahash M, Susin C, Polimeni G, Hall J, Wikesjö UM. Bone healing dynamics at buccal peri-implant sites. Clin Oral Implants Res 2008;19:166-72.

8. Coatoam GW, Mariotti A. The segmental ridge-split procedure. J Periodontol 2003;74:757-70.

9. Shimoyama T, Kaneko T, Shimizu S, Kasai D, Tojo T, Horie, N. Ridge widening and immediate implant placement: a case report. Implant Dent 2001;10:108-12. 